\title{
Development and Validation a Nomogram and Prognosis of Chemotherapy for Evaluation in Giant- cell Lung Carcinoma With Metastases: A Propensity Score Matching Analysis
}

\section{Kun Chen \\ Wenzhou Medical University \\ Xinbo Zhang \\ Wenzhou Medical University \\ Tianhao Zhao \\ Wenzhou Medical University \\ Deyao Xie \\ Wenzhou Medical University \\ Xiaofang Deng \\ Wenzhou Medical University \\ Jiandong Zhang \\ Wenzhou Medical University \\ Dezhi Cheng \\ Wenzhou Medical University}

Zhihao Yang ( $\mathbf{3 4 7 8 0 3 3 7 8 7 @ q q . c o m ~ ) ~}$

Wenzhou Medical University https://orcid.org/0000-0002-1924-9987

\section{Research}

Keywords: Giant Cell Lung Carcinoma, Chemotherapy, Nomogram, Forest Plot, Propensity Score Matching Analysis

Posted Date: June 19th, 2020

DOI: https://doi.org/10.21203/rs.3.rs-35419/v1

License: (c) (1) This work is licensed under a Creative Commons Attribution 4.0 International License.

Read Full License 


\section{Abstract}

Background and Objectives: Whether chemotherapy couldimprove prognosis for giant-celllung carcinoma with metastases remains controversial. The present study aimed to determine the significanceof chemotherapy in patients with metastases giant-cell Lung carcinoma, and to develop a nomogram to predict its outcomes.

Methods:Data of 566 patients from the Surveillance, Epidemiology, and End Results(SEER) database were analyzed; 346 matched patients were divided into a chemotherapy group and non-chemotherapy group, respectively, using the propensity scorematching method. Univariate and multivariate analyses were performed to determine theprognostic factors of giant-celllung carcinoma, and subgroup analysis was performed according tothe site of metastases. While a visual nomogram wasestablished to judge the prognosis.

Results: With the median follow-up of 20.5 months, The 3-yearsurvival rates were $20.2 \%$ in the chemotherapy set and $16.7 \%$ in the non-chemotherapy set $(\mathrm{P}=0.006)$.Chemotherapy did improve the Giant-cell Lung Carcinoma survival rates in patients with metastases $(\mathrm{HR}=0.490,95 \% \mathrm{Cl}=0.358-0.669$, $\mathrm{P}<0.001)$. In subgroup analysis, radiotherapy did not improved the Giant-cell Lung Carcinoma survival rates in patients with metastases $(\mathrm{HR}=0.951,95 \% \mathrm{Cl}=0.690-1.311, \mathrm{P}=0.758$, Table 2). Chemotherapy improved the CSS in patients with lung metastases $(\mathrm{HR}=0.090,95 \% \mathrm{Cl}=0.017-0.470, \mathrm{P}=0.004) . \mathrm{In}$ addition, chemotherapy improved the CSS in patients with brain metastases $(\mathrm{HR}=0.117,95 \% \mathrm{Cl}=0.014$ $0.955, P=0.045$ ). Compared with bone metastasis, liver metastasis and metastasis of more than 2 sites, the effect of chemotherapy is not obvious.Furthermore, our nomogram could predict the probability of surviving to the median survival time of the population with a c-index of 0.768 .

Conclusion:The benefit of chemotherapy in giant-celllung carcinoma with metastases patients was obviously different, andthe recommendation of chemotherapy for this population should be individualized. chemotherapy shouldbe considered for patients with only lungmetastasis or brain metastasis, but chemotherapy could be avoided in those with other site metastases or multiple metastases. We developed the first competing risk nomogram to predict the risk of GCLC patients, which performed well in the evaluation and might be helpful for individualized screening.

\section{Introduction}

Lung cancer accounts for a relatively large proportion of human tumors around the world and bring about approximately $30 \%$ of tumor-related deaths. Most lung cancers are non-small cell tumors. This type accounts for nearly $80 \%$ of all carcinoma of the lungs, and only 4-6 percent of patients were alive more than five years[1]. GCLC is a poorly differentiated carcinomaconsists of highly polymorphic mononuclear or multinuclear giant cells and is identified as one of the non-small cell lung carcinoma (NSCLC)[2]. A studydescribed that GCLC accounted for nearly $0.3-0.4$ percent of primary lung cancers, with an growth rate of approximately three new cases per million persons per year among the 60,000 patients in the 
US[3]. GCLC appears as a large necrotic block with no obvious structural form. Microscopic observation shows that GCLC comprises giant, multinucleated, and weirdly shaped cells. Its background contains a large number of neutrophils and lymphocytes, and there is no specific pattern of adenocarcinoma, squamous cell carcinoma, or large squamous cell carcinoma[4]. Five subsets were identified by World Health Organization (WHO) in 2004: spindle cell carcinoma,pleomorphic carcinoma, giant cell carcinoma,pulmonary blastoma and carcinosarcoma[2]. Currently, we can determine that a tumor is GCLC by using $\mathrm{CT}$, which shows a large and central low-attenuation area or cavity. This imaging is consistent with the tumor necrosis area found in pathological specimens[5]. There are data showing that the palliative chemotherapy response in advanced lung sarcomatoid carcinoma is poor[6]. Patients who do not undergo surgery after receiving appropriate chemotherapy, radiotherapy or combined adjuvant therapy can also have a prolonged survival time[7]. Because the poor prognosis and shortened survival time associated with tumour heterogeneity is worse than that for ordinary NSCLC, Maneenil et al recommend early consideration of surgical resection and perioperative chemotherapy[8].

A nomogram is an intuitive diagram to quantify and provide the survival probability of a GCLC patients undergoing the progress of clinical events $[9,10]$.The thoracic surgeon can refer to such a probability to make a decision on the next treatment plan.In addition, we not only assessed the accuracy of the nomogram, but also calibrated it.

\section{Methods}

\section{Patient selection}

This retrospective study was conducted by acquiring data from the Surveillance Epidemiology and End Results (SEER) database. Data were obtained by SEER`Stat8.3.6 in October 2019. Using this software, wescreened GCLC patients between 2004 and 2016. We limitedthisstudypopulation based onthe followingcriteria:age at diagnosis: $\geq 18$ years old,primary site:lung and bronchus, primary site and morphology ICD-0-3: GCLC (8030,8031,8032,8033,8034, and 8035). Patients were excluded if their age, race, survival time, pathologicaldiagnosis, or presence/absence of metastasis wereunknown or if their pathological results were from an autopsy or a death certificate.

Forthe SEERdatabase, cancer-specific survival (CSS) was defined as death due to GCLC, and overall survival (OS) was defined as deathregardless of cause. The primary outcome was CSS, with OS and CSS considering other non-GCLC-relateddeaths as the secondary outcome. Survival time was defined as the time from diagnosis to the date of death.

\section{Ethics statement}

Data from theSEERdatabase are free to use and donotrequireinformedpatientconsent. Thepresentstudycomplied withthe1964HelsinkiDeclarationanditslateramendmentsorcomparableethicalstandards. It was not 
appropriate or possible to involve patients or the public in the design, or conduct, or reporting, or dissemination plans of research

\section{Statistical analysis}

Clinical characteristics were compared among thechemotherapy set and nonchemotherapy set, using Pearson's chi-square test. To remove confounding factors between baseline characteristics among each set as much as possible, we set the proportion for propensity score matching (PSM) analysis at0.2. CSS analysis was conducted after PSM,and survival curves were created for age,race,T stage,N stage,M stage,chemotherapy, surgery and radiotherapy by using the Kaplan-Meier method. The CSS valuesbetween the different sets were compared by performing the log-rank test. Univariate and multivariate Cox regression models were used to evaluate the CSS hazard ratios (HRs) and 95\% confidence intervals (Cls) of patients in the chemotherapy set compared to those in the nonchemotherapy set. In the univariate analysis, we added meaningful or clinical variables to the multivariate Cox regression analyses. HRs and $95 \% \mathrm{Cls}$ were calculated by using multivariate Cox proportion hazard models, with adjustments for age, race, sex, T stage, $\mathrm{N}$ stage, $\mathrm{M}$ stage, grade, chemotherapy, surgery and radiotherapy. Multivariate Cox models were conducted for subset analyses to determine whether there was a significant interaction between different characteristics. A novel visible nomogram of the model was developed to evaluate the discriminatory performances, which was measured by the concordance index (C-index) , and the C-index was adjusted for the predictive accuracy, which was equaled to the area under curve of receiver operating characteristic curves. The calibration curve of nomogram was setting to reflect the relationships of the predicted out versus observed results. All statistical analyses for predicting CSS were performed using $R$ software (version 3.6.3). All $P$ values lower than 0.05 with two-sided comparisons were deemed to indicate statistical significance.

\section{Results}

\section{Tumour information and patient characteristics}

A total of 566 patients withGCLC who met the research criteria were eventually selected. The median age of patients was 71 years (interquartile range (IQR): 63-78). Tumour information and patient characteristics stratified by chemotherapy are summarized in Table 1 . There were significant differences in age at diagnosis, $\mathrm{T}$ stage, $\mathrm{N}$ stage, $\mathrm{M}$ stage and surgery between the chemotherapy set and the nonchemotherapy set $(P<0.001)$. The PSMmethodwas used to balance baseline characteristics between each set, and there were 173 patients in each set after PSM. Between the two sets, we found that T stage, $\mathrm{N}$ stage, $\mathrm{M}$ stage and surgery showed statistically significant differences $(P<0.05)$; for other baseline characteristics, no significant differences were observed $(P>0.05$, Table1).

\section{Cancer-specific survival analysis}

The median survival was 20.5 months (IQR: 17.8-22.5), and 188 people died of GCLC. There were 93 (26.88\%) GCLC-related death events observed in the chemotherapy set and 95 (27.46\%) in the 
nonchemotherapy set. The 3-year CSS were $20.2 \%$ in the chemotherapy set and $16.7 \%$ in the nonchemotherapy set $(P=0.006)$. Kaplan-Meier analysis showed that patients treated with chemotherapy had a better CSS than patients who did not receive chemotherapy; the log-rank test $P$ value was 0.037 (Figure 1A). In the univariate analyses,age, race, T stage, $\mathrm{N}$ stage, $\mathrm{M}$ stage, chemotherapy, surgery and radiotherapy were significantly associated with CSS. Except for the radiotherapy results, the results from the multivariate analyses were consistent of univariate analyses; the details are shown in Table 2. In the multivariate Cox regression analyses, radiotherapy did not significantly improve the CSS for GCLC patients (HR $=0.951,95 \% \mathrm{Cl}=0.690-1.311, \mathrm{P}=0.758$, Table 2). Cox proportional hazard regression analyses revealed that $\mathrm{T}$ stage, $\mathrm{N}$ stage, metastatic sites and surgery were independent prognostic factors. Age, grade, race, sex and radiotherapy were not significant factors. As shown in Table 3 , chemotherapy improved the CSS in patients with lung metastases $(\mathrm{HR}=0.090,95 \% \mathrm{Cl}=0.017-0.470, \mathrm{P}$ $=0.004$, Figure 1B, Table 3). In addition, chemotherapy improved the CSS in patients with brain metastases $(\mathrm{HR}=0.117,95 \% \mathrm{Cl}=0.014-0.955, \mathrm{P}=0.045$, Figure $1 \mathrm{E}$, Table 3$)$. Surprisingly, we found that there were no differences in the CSSs in patients with no metastases or with multiple metastases(Figure 1C,Figure 1D,Figure 1F). The results are shown in a forest plot to make them more intuitive (Figure 2).

\section{Nomogram}

Eventually, in order to better estimate the multiple independent risk factors, which were according to univariate and multivariate COX regression analyses, of CCS in GCLC patients for prognostic capability(Figure 3).To interpret the meaning of graph, we set a patient's value is located on each variable axis, then depict a red line to identify the number of points for which we choose the variable. The integrated all points is positioned to the total points axis, which would determine the likelihood of median survivaltime for 1 or 3 years by a red line was drawn downward. The gray color area under the cure of total points and the green box represent the sample size, which shows the patients statistics of the GCLC and the populationdistribution of the prognosis. We found that the prediction ofC-index was $0.768[95 \%$ confidence interval $(\mathrm{Cl}), 0.746-0.789]$. The Receiver Operating Characteristic (ROC) was used to evaluate the accuracy of the model(Figure 4 ), The Area Under Curve (AUC) is $0.877(95 \%$ confidence interval $(\mathrm{Cl}), 0.835-0.919)$. The calibration plot of the nomogram had reached a good concordance between the CSS prediction risk and the actual observed incidence(Figure 5).

\section{Discussion}

With the improvement of diagnostic techniques and treatment options, the OS and CSS rates of patients with non-metastatic NSCLC continue to increase. However, in terms of metastasis, the time from the occurrence of the primary tumor to the determination of metastasis is not certain. It is generally believed that this process requires a certain amount of time for the appropriate conditions to develop and interactions within the tumor microenvironment. Altorki et al's research shows that before metastasis, immune cells[11], organ-specific inducing substances, growth factors, inflammatory factors and extracellular matrix-modified proteins create a more favourable microenvironment for metastatic tumour cells. The method by which metastatic tumour cells interact with the host organ microenvironment is 
complex. Different types of interactions may lead to unique patterns of transfer events. The Vieira et al study found that most patients with lung sarcomatoid carcinoma have a high vascular invasion rate and a high recurrence rate after surgical resection, which indicates that this type of tumour has a high metastasis rate and poor prognosis characteristics $[12,13]$. In previous research, we found that approximately $71.9 \%$ of patients with lung sarcomatoid carcinoma are smokers. Such a high smoking rate is related to the occurrence of tumours, but smoking status has no significant effect on the prognosis and survival of patients with lung cancer[12,14]. At the same time, the formulation of chemotherapy programmes has been reported for different lung cancer subtypes and stages, and the effects of single and combined targeted therapy applications have also been reported[15-17]. We studied the effect of chemotherapy on the CSS rate of GCLC through PSM. Some of the clinicopathological parameters showed significant differences between the chemotherapy set and the nonchemotherapy set. It was found that patients younger than 71 years old had a good prognosis after chemotherapy, and patients older than 71 years old had no difference in prognosis, regardless of whether they received chemotherapy or not; this may be related to the body's cardiopulmonary functional reserve, the tolerance to toxicity and the side effects of chemotherapy drugs. It is reported that the proportion of patients aged 80 and above who undergo systemic chemotherapy has decreased significantly, which is consistent with our previous PSM data. There were 184 people who were 71 years old or older and did not receive chemotherapy. The possible reason is that elderly patients and their families may be unwilling to undergo this treatment. Therefore, due to the side effects, we prefer to suggest a milder treatment for advanced cancer in elderly patients[18]. There are also studies showed that few elder patients receive further treatment after relapse or metastasis[19]. Our data from the PSM analysis show that T stage, $\mathrm{N}$ stage, metastatic factors, surgery and chemotherapy are statistically significant factors, but the differences in outcomes related to chemotherapy, race and sex are not obvious. Interestingly, there was no significant difference in the effect of radiotherapy in our study, either within or between sets. Martin et al's study showed that the benefits of postoperative radiotherapy have not been shown to improve the survival rate of patients with typical lung sarcomatoid carcinoma[20]. According to previous research, platinum-based palliative chemotherapy strategies are less effective for the treatment of lung cancer, and the results for GCLC as a subtype in our study are slightly different from those in this previous report. We found that patients receiving chemotherapy had improved CSS compared with that of patients not receiving chemotherapy. An analysis within the chemotherapy set showed that patients with lung,brain, and bone metastases had a significantly better CSS than those in the nonchemotherapy set. The analysis between sets showed that chemotherapy had no significant effect on the prognosis of patients with or without metastasis. A possible reason is that the systemic chemotherapy does not convey additional benefits in patients without metastasis. Local treatments, including stereotactic body radiation therapy (SBRT), and other treatments, can also be utilized. A multicentre, randomized, controlled phase 2 study showed that local consolidation therapy in oligometastatic NSCLC can significantly prolong the survival duration while delaying the appearance of new metastatic foci[21].Patients with single-site metastases with only brain or intra-pulmonary metastases have better CSS with chemotherapy.Previous studies have many controversial aspects, and one study showed that palliative chemotherapy used in NSCLC is not effective for advanced lung sarcomatoid carcinoma. No patients achieved an effective response after 
chemotherapy, and the median OS was only 5 months. Lung sarcomatoid carcinoma has a higher rate of local recurrence after surgery and a higher incidence of metastases at diagnosis[6]. The Vieira et al study showed that patients receiving platinum chemotherapy had a prolonged OS while with no difference in PFS[22]. Chaft et al's research suggests that not all lung sarcomatoid carcinomas are refractory to chemotherapy. Due to the poor prognosis after recurrence of sarcomatoid carcinoma, relapse should be prevented and delayed as much as possible. Neoadjuvant or adjuvant chemotherapy could be used in patients with resectable lung cancer. In addition, according to Wang et al's research, when NSCLC has a high degree of vascular invasion, the relative risk of recurrence and death is 4 and 2 times higher. Therefore, it is recommended that NSCLC patients receive systemic chemotherapy rather than palliative chemotherapy[23,24]. However, the analysis within the set showed that the prognosis of patients with liver metastases was worse than that of the other metastatic sets[25], and the effect of chemotherapy was poor. The results of the Wu et al study identified that patients with liver metastases had significantly lower PFS and OS. Rong et al showed that the effect of chemotherapy in early bone metastasis was significant; however, in this retrospective analysis, the effect of chemotherapy on bone metastasis was not beneficial as expected. The analysis between sets showed that chemotherapy had no significant effecton patients with 2 or more metastases. The possible reason is thatan increased tumor burden leads to chemotherapy being ineffective. The Joss study found that patients with two or more metastases in distant organs had a lower chemotherapy response rate than patients with less tumor burden (patients with local disease or a metastatic disease in an extrathoracic organ); the reason may be that a larger tumor burden results in more cell populations that are resistant to multiple drugs[26].

Overall, the prognosis of GCLC patients is unsatisfactory. At present, an army of clinical prediction models are showing a hot trend, but most of the clinical prediction models are general studies of NSCLC, and do not involve the prognosis prediction of GCLC[27-29].In order to express this screening and integration data more concretely, we constructed this nomogram for clinical practice conveniently, which predicted its 1 and 3 year survival probability. Contemporarily, with the sustainable basic study and the development of cure strategies[30,31], and the novel detection techniques appeared[32],The new prediction model we have established opens up a novel way for the prognosis prediction of GCLC patients through the independent prognostic factors screened. The evaluation of this model has a well accuracy and calibration. The C-index value for predicting the survival probability was 0.768 , The Area Under Curve of ROC was 0.877 , which was statistically higher than that TNM $8^{\text {th }}$ Edition[33].

The strange thing is that radiotherapy is not included in the nomogram model. We suspect that this may be related to the dose and location of radiotherapy. According to Johnson's point of view, the increase in dose compared with the standard dose of radiotherapy can improve the survival rate. A high dose of cardiac radiotherapy is an important independent prognostic factor to reduce survival rate[34].

Our research still has many shortcomings. For example, the relationship between chemotherapy and the time of surgery in the SEER database is not clear; second, the description of the type of surgery is slightly unclear, and it is not clear whether the surgery performed was a lobectomy or lung segment resection. In addition, data on complications and costs of treatment are minimal, the sample size for tumor data is 
small, and the tracking time is short. The abovementioned limitations may introduce a degree of uncertainty in our results. The data in the SEER database are common, and it lacks the unique data contained in databases in other regions, but the SEER database does provide many types of valid and reliable data for retrospective analysis. Our data compilation is expected to provide a better treatment results for patients with GCLC.

\section{Conclusion}

In summary, we found that $\mathrm{T}$ stage, $\mathrm{N}$ stage, metastatic site, chemotherapy and surgery were associated with the CSSof GCLC.Patient characteristics has little effect on prognosis. In future research we propose exploring the effect of tumour heterogeneity on prognosis. The effect of radiotherapy fortreating GCLC was not a significant factor before and after matching; therefore, it is recommended that this treatment should not be considered as the primary method. The benefit of chemotherapy in patients with GCLC varies greatly, and the most rational proposal of chemotherapy for this population should be individualized. Patients with only brain or lung metastases may undergo chemotherapy, but chemotherapy could be avoided in those with no metastases or multiple metastases. This study development an ideal model of nomogram, which predict the survival outcomes for GCLC patients consultation and thoracic surgeon making a reasonable decision. These resultsenhance our understanding of GCLC and highlight how cancertreatment strategies can be improved.

\section{Declarations}

\section{Acknowledgments}

I thank Dr. Dezhi Cheng and Dr. Deyao Xie (Wenzhou Medical University凶China) for their review of the article.

\section{Authors' contributions}

Kun Chen and Zhihao Yang collected the data and drafted the manuscript. Dezhi Cheng conceived and designed the study and reviewed and finalized the manuscript. All authors read and approved the final manuscript.

\section{Funding}

Not applicable.

\section{Availability of data and materials}

The datasets used and analyzed during the current study are available from the Surveillance, Epidemiology, and End Results(SEER) database.

\section{Ethics approval and consent to participate}


Not applicable.

\section{Consent for publication}

Not applicable.

\section{Competing interests}

The authors declare that they have no competing interests.

\section{Author details}

${ }^{1}$ The First Affiliated Hospital of Wenzhou Medical University,Zhejing,325000,China. ${ }^{2}$ The First Affiliated Hospital of Wenzhou Medical University,Zhejing,325000,China. ${ }^{3}$ The First Affiliated Hospital of Wenzhou Medical University,Zhejing,325000,China. ${ }^{4}$ The First Affiliated Hospital of Wenzhou Medical University,Zhejing,325000,China. ${ }^{5}$ The First Affiliated Hospital of Wenzhou Medical University,Zhejing,325000,China. ${ }^{6}$ The First Affiliated Hospital of Wenzhou Medical University,Zhejing,325000,China. ${ }^{7}$ The First Affiliated Hospital of Wenzhou Medical University,Zhejing,325000,China. ${ }^{8}$ The First Affiliated Hospital of Wenzhou Medical University,Zhejing,325000,China.

\section{References}

1. Bray, F., et al., Global cancer statistics 2018: GLOBOCAN estimates of incidence and mortality worldwide for 36 cancers in 185 countries. CA: A Cancer Journal for Clinicians, 2018. 68(6): p. 394424.

2. E, B., et al., The new World Health Organization classification of lung tumours. 2001. 18(6): p. 105968.

3. WD, T., T. LB, and D.S.J. Cancer, Lung cancer. 1995. 75: p. 191-202.

4. Hiroshima, K., et al., Cytological characteristics of pulmonary pleomorphic and giant cell carcinomas. Acta Cytol, 2011. 55(2): p. 173-9.

5. Fujisaki, A., et al., Pleomorphic Carcinoma of the Lung: Relationship Between CT Findings and Prognosis. AJR Am J Roentgenol, 2016. 207(2): p. 289-94.

6. Bae, H.M., et al., Palliative chemotherapy for pulmonary pleomorphic carcinoma. Lung Cancer, 2007. 58(1): p. 112-5.

7. K, I., et al., Clinical characteristics of pleomorphic carcinoma of the lung. 2010. 68(2): p. 204-10.

8. G, R., et al., Pulmonary carcinomas with pleomorphic, sarcomatoid, or sarcomatous elements: a clinicopathologic and immunohistochemical study of 75 cases. 2003. 27(3): p. 311-24.

9. P, H., et al., Transparent Reporting of Multivariable Prediction Models in Journal and Conference Abstracts: TRIPOD for Abstracts. 2020. 
10. A, I., et al., How to build and interpret a nomogram for cancer prognosis. 2008. 26(8): p. 1364-70.

11. NK, A., et al., The lung microenvironment: an important regulator of tumour growth and metastasis. 2019. 19(1): p. 9-31.

12. Maneenil, K., et al., Sarcomatoid Carcinoma of the Lung: The Mayo Clinic Experience in 127 Patients \%J Clinical Lung Cancer. 2018. 19(3).

13. Vieira, T., et al., Blood vessel invasion is a major feature and a factor of poor prognosis in sarcomatoid carcinoma of the lung \%J Lung Cancer. 2014. 85(2).

14. Gu, L., et al., Clinical analysis of 95 cases of pulmonary sarcomatoid carcinoma \%J Biomedicine \& Pharmacotherapy. 2015. 76.

15. X, Y., et al., Efficacy of first-line treatment with epidermal growth factor receptor-tyrosine kinase inhibitor (EGFR-TKI) alone or in combination with chemotherapy for advanced non-small cell lung cancer (NSCLC) with low-abundance mutation. 2019. 128: p. 6-12.

16. J, W., et al., Should patients with stage IB non-small cell lung cancer receive adjuvant chemotherapy? A comparison of survival between the 8th and 7th editions of the AJCC TNM staging system for stage IB patients. 2019. 145(2): p. 463-469.

17. VF, V., et al., Cisplatin versus carboplatin in combination with third-generation drugs for advanced non-small cell lung cancer. 2020. 1: p. CD009256.

18. CL, H., et al., Advanced non-small cell lung cancer in the elderly: the impact of age and comorbidities on treatment modalities and patient prognosis. 2015. 6(1): p. 38-45.

19. ML, W., et al., Impact of age and comorbidity on treatment of non-small cell lung cancer recurrence following complete resection: A nationally representative cohort study. 2016. 102: p. 108-117.

20. JY, H., et al., The role of palliative chemotherapy for advanced pulmonary pleomorphic carcinoma. 2009. 26(3): p. 287-91.

21. DR, G., et al., Local consolidative therapy versus maintenance therapy or observation for patients with oligometastatic non-small-cell lung cancer without progression after first-line systemic therapy: a multicentre, randomised, controlled, phase 2 study. 2016. 17(12): p. 1672-1682.

22. Vieira, T., et al., Efficacy of First-Line Chemotherapy in Patients with Advanced Lung Sarcomatoid Carcinoma \% J Journal of Thoracic Oncology. 2013. 8(12).

23. J, W., et al., Blood vessel invasion as a strong independent prognostic indicator in non-small cell lung cancer: a systematic review and meta-analysis. 2011. 6(12): p. e28844.

24. Martin, L.W., et al., Sarcomatoid Carcinoma of the Lung: A Predictor of Poor Prognosis \% J The Annals of Thoracic Surgery. 2007. 84(3).

25. KL, W., et al., Liver metastasis predicts poorer prognosis in stage IV lung adenocarcinoma patients receiving first-line gefitinib. 2015. 88(2): p. 187-94.

26. RA, J., et al., Combination chemotherapy with mitomycin, vindesine, and cisplatin for non-small cell lung cancer. Association of antitumor activity with initial tumor burden and treatment center. 1990. 65(11): p. 2426-34. 
27. W, L., et al., Development and validation of a nomogram for predicting survival in patients with resected non-small-cell lung cancer. 2015. 33(8): p. 861-9.

28. J, W., et al., Nomogram integrating gene expression signatures with clinicopathological features to predict survival in operable NSCLC: a pooled analysis of 2164 patients. 2017. 36(1): p. 4.

29. O, B., et al., Long-term survival after non-small cell lung cancer surgery: development and validation of a prognostic model with a preoperative and postoperative mode. 2006. 132(3): p. 491-8.

30. Domblides, C., et al., Efficacy of Immune Checkpoint Inhibitors in Lung Sarcomatoid Carcinoma \%J Journal of Thoracic Oncology. 2020.

31. L, X., S. YW, and X.Y.J.J.o.t.o.o.p.o.t.I.A.f.t.S.o.L. Cancer, Nkx2-4 Mutation Confers Resistance to EGFR-Tyrosine Kinase Inhibitors in EGFR-Mutant Lung Sarcomatoid Carcinoma. 2019. 14(6): p. e125e126.

32. X, Z., et al., The Precise Diagnosis of Cancer Invasion/Metastasis via 2D Laser Ablation Mass Mapping of Metalloproteinase in Primary Cancer Tissue. 2018. 12(11): p. 11139-11151.

33. Y, Z., et al., A Nomogram for Predicting Cancer-Specific Survival of TNM 8th Edition Stage I Nonsmall-cell Lung Cancer. 2019. 26(7): p. 2053-2062.

34. MD, J., et al., Matched-Pair Analysis of High Dose Versus Standard Dose Definitive Chemoradiation for Locally Advanced Non-Small-Cell Lung Cancer. 2017. 18(2): p. 149-155.

\section{Tables}




\begin{tabular}{|c|c|c|c|c|c|c|c|}
\hline \multicolumn{8}{|c|}{$\begin{array}{l}\text { Table1 Comparison o } \\
\text { ho-CHEMO group. }\end{array}$} \\
\hline \multirow{2}{*}{\multicolumn{2}{|c|}{ Variables }} & \multicolumn{2}{|c|}{ Before PSM } & \multirow[t]{3}{*}{ P-value } & \multicolumn{2}{|l|}{ After PSM } & \multirow[t]{3}{*}{ P-value } \\
\hline & & CHEMO & No-CHEMO & & CHEMO & No-CHEMO & \\
\hline & & $N=261$ & $\mathrm{~N}=305$ & & $N=173$ & $\mathrm{~N}=173$ & \\
\hline \multirow[t]{2}{*}{ AGE } & $\leqslant 71$ & 177 & 121 & 0.035 & 97 & 89 & 0.101 \\
\hline & $>71$ & 84 & 184 & & 76 & 84 & \\
\hline \multicolumn{2}{|c|}{ GRADE 1} & 3 & 4 & 0.340 & 3 & 2 & 0.584 \\
\hline & 2 & 1 & 2 & & 0 & 1 & \\
\hline & 3 & 195 & 234 & & 126 & 129 & \\
\hline & 4 & 62 & 65 & & 44 & 41 & \\
\hline \multirow[t]{3}{*}{ RACE } & White & 221 & 258 & 0.338 & 148 & 141 & 0.103 \\
\hline & Black & 22 & 37 & & 14 & 26 & \\
\hline & Other & 18 & 10 & & 11 & 6 & \\
\hline \multirow[t]{2}{*}{ SEX } & Male & 157 & 183 & 0.807 & 100 & 105 & 0.581 \\
\hline & Female & 104 & 122 & & 73 & 68 & \\
\hline \multirow[t]{4}{*}{$T$} & 1 & 13 & 35 & $<0.001$ & 11 & 14 & 0.001 \\
\hline & 2 & 84 & 91 & & 60 & 53 & \\
\hline & 3 & 89 & 109 & & 57 & 72 & \\
\hline & 4 & 74 & 70 & & 45 & 34 & \\
\hline \multirow[t]{4}{*}{$N$} & 0 & 113 & 185 & $<0.001$ & 89 & 95 & $<0.001$ \\
\hline & 1 & 38 & 34 & & 26 & 19 & \\
\hline & 2 & 84 & 66 & & 44 & 42 & \\
\hline & 3 & 26 & 20 & & 14 & 17 & \\
\hline \multirow[t]{6}{*}{$M$} & None & 176 & 210 & $<0.001$ & 114 & 115 & 0.001 \\
\hline & Bone & 19 & 19 & & 16 & 13 & \\
\hline & Brain & 16 & 15 & & 5 & 11 & \\
\hline & Liver & 2 & 5 & & 2 & 3 & \\
\hline & Lung & 13 & 21 & & 12 & 9 & \\
\hline & Multiple & 35 & 34 & & 24 & 22 & \\
\hline \multirow[t]{2}{*}{ SURG } & No & 163 & 175 & $<0.001$ & 101 & 102 & $<0.001$ \\
\hline & Yes & 98 & 130 & & 72 & 71 & \\
\hline \multirow[t]{2}{*}{ RADIO } & No & 122 & 232 & 0.340 & 117 & 109 & 0.849 \\
\hline & Yes & 139 & 73 & & 56 & 64 & \\
\hline
\end{tabular}




\begin{tabular}{|c|c|c|c|c|c|}
\hline \multirow{3}{*}{\multicolumn{2}{|c|}{$\begin{array}{r}\text { Table2 } \\
\text { Variables }\end{array}$}} & \multicolumn{2}{|c|}{ Univariate analysis } & \multicolumn{2}{|c|}{ Multivariate analysis } \\
\hline & & $\mathrm{HR}$ & D & $\mathrm{HR}$ & $P$ \\
\hline & & (95\% Cl) & r & $(95 \% \mathrm{Cl})$ & $r$ \\
\hline \multirow[t]{2}{*}{ AGE } & $\leqslant 71$ & Reference & & Reference & - \\
\hline & $>71$ & $1.309(1.034-1.659)$ & 0.026 & $0.766(0.556-1.056)$ & 0.104 \\
\hline \multirow[t]{3}{*}{ GRADE } & $1+11$ & Reference & . & Reference & - \\
\hline & $\| H+N$ & $1.364(0.563-3.306)$ & 0.491 & 0.1 .219 & 0.784 \\
\hline & m. & $1.304,0.305=3.50 \%$ & 0.75 & $(0.297-5.002)$ & \\
\hline \multirow[t]{3}{*}{ RACE } & White & Reference & - & Reference & - \\
\hline & Bladk & $1.475(1.046-2.081)$ & 0.027 & $1.408(0.917-2.160)$ & 0.118 \\
\hline & Other & $0.859(0.491-1.502)$ & 0.594 & $1.755(0.885-3.480)$ & 0.107 \\
\hline \multirow[t]{2}{*}{ SEX } & Male & Reference & & Reference & - \\
\hline & Female & $0.951(0.748-1.208)$ & 0.679 & $1.133(0.831-1.546)$ & 0.430 \\
\hline \multirow[t]{7}{*}{$T$} & 1 & Reference & - & Reference & - \\
\hline & 2 & $2.739(1.420-5.283)$ & 0.003 & 5.084 & 0.002 \\
\hline & 2 & & & $(1.822-14.183)$ & \\
\hline & 3 & $4.415(2.304-8.459)$ & $<0.001$ & 7.556 & $<0001$ \\
\hline & & & & $(2.731-20.909)$ & \\
\hline & 4 & 6.229 & $<0.001$ & 6.545 & 0001 \\
\hline & & (3.196-12.141) & & (2.301-18.614) & 0001 \\
\hline \multirow[t]{4}{*}{$N$} & $\mathbf{0}$ & Reference & & Reference & - \\
\hline & 1 & $1.939(1.356-2.781)$ & $<0.001$ & $1.924(1.209-3.062)$ & 0.006 \\
\hline & 2 & $2.691(2.025-3.577)$ & $<0.001$ & $1.846(1.251-2.724)$ & 0.002 \\
\hline & 3 & $3.563(2.380-5.336)$ & $<0.001$ & $2.233(1.339-3.722)$ & 0.002 \\
\hline \multirow[t]{3}{*}{ M } & None & Reference & . & Reference & . \\
\hline & Single & $2.717(2.033-3.630)$ & $<0.001$ & $1.863(1.250-2.777)$ & 0.002 \\
\hline & Multiple & $4.582(3.222-6.517)$ & $<0.001$ & $2.736(1.730-4.327)$ & $<0001$ \\
\hline \multirow[t]{2}{*}{ SURG } & No & Reference & - & Reference & - \\
\hline & Yes & $0.273(0.209-0.356)$ & $<0.001$ & $0.380(0.249-0.582)$ & $<0001$ \\
\hline \multirow[t]{2}{*}{ RADIO } & No & Reference & - & Reference & - \\
\hline & Yes & $1.443(1.133-1.838)$ & 0.003 & $0.951(0.690-1.311)$ & 0.758 \\
\hline \multirow[t]{2}{*}{ CHEMO } & No & Reference & & Reference & - \\
\hline & Yes & $0.744(0.559-0.991)$ & 0.043 & $0.490(0.358-0.669)$ & $<0001$ \\
\hline
\end{tabular}




\begin{tabular}{|c|c|c|c|c|}
\hline Subgroup & $\begin{array}{c}\text { Chemolpatients/even } \\
\text { ts) }\end{array}$ & \begin{tabular}{|c|} 
No \\
Chemo(patients/even \\
ts) \\
\end{tabular} & Hazard Ratio(95\%Cl) & $P$ \\
\hline \multicolumn{5}{|l|}{ Age } \\
\hline Less than $71 \mathrm{yr}$ & $186 / 53$ & $186 / 54$ & $0.655(0.447-0.958)$ & 0.029 \\
\hline More than $71 \mathrm{yr}$ & $160 / 40$ & $160 / 41$ & $0.891(0.576-1.378)$ & 0.604 \\
\hline \multicolumn{5}{|l|}{ Race } \\
\hline White & $289 / 77$ & $289 / 71$ & $0.796(0.576-1.100)$ & 0.166 \\
\hline Black & $40 / 11$ & $40 / 19$ & $0.835(0.397-1.757)$ & 0.634 \\
\hline Other & $17 / 5$ & $17 / 5$ & $0.410(0.116-1.448)$ & 0.166 \\
\hline \multicolumn{5}{|l|}{ Sex } \\
\hline Male & $205 / 57$ & $205 / 59$ & $0.785(0.545-1.130)$ & 0.193 \\
\hline Female & $141 / 36$ & $141 / 36$ & $0.681(0.428-1.082)$ & 0.104 \\
\hline \multicolumn{5}{|l|}{ Grade } \\
\hline $1+11$ & $6 / 2$ & $6 / 0$ & $0.744(0.559-0.991)$ & 0.043 \\
\hline$I I I+N$ & $340 / 91$ & $340 / 95$ & $0.726(0.544-0.969)$ & 0.029 \\
\hline \multicolumn{5}{|l|}{$T$} \\
\hline $\mathrm{T} 1$ & $25 / 2$ & $25 / 2$ & $1.741(0.243-12.445)$ & 0.581 \\
\hline $\mathrm{T} 2$ & $113 / 30$ & $113 / 23$ & $0.983(0.577-1.713)$ & 0.983 \\
\hline $\mathrm{T} 3$ & $129 / 35$ & $129 / 46$ & $0.631(0.405-0.982)$ & 0.041 \\
\hline $\mathrm{T} 4$ & $79 / 26$ & $79 / 24$ & $0.310(0.170-0.566)$ & $<0.001$ \\
\hline \multicolumn{5}{|l|}{ N } \\
\hline No & $184 / 36$ & $184 / 37$ & $0.929(0.587-1.471)$ & 0.753 \\
\hline N1 & $45 / 18$ & $45 / 10$ & $0.827(0.379-1.807)$ & 0.635 \\
\hline N2 & $86 / 29$ & $86 / 34$ & $0.350(0.207 \cdot 0.592)$ & $<0.001$ \\
\hline N3 & $31 / 10$ & $31 / 14$ & $0.305(0.123-0.759)$ & 0.011 \\
\hline \multicolumn{5}{|l|}{ Surge } \\
\hline Yes & $143 / 27$ & $143 / 27$ & $0.853(0.500-1.455)$ & 0.56 \\
\hline No & $203 / 66$ & $203 / 68$ & $0.599(0.426-0.843)$ & 0.003 \\
\hline \multicolumn{5}{|l|}{ Radio } \\
\hline Yes & $120 / 39$ & $120 / 39$ & $0.836(0.536-1.305)$ & 0.431 \\
\hline No & $226 / 54$ & $226 / 56$ & $0.699(0.480-1.017)$ & 0.061 \\
\hline \multicolumn{5}{|l|}{$M$} \\
\hline None & $229 / 50$ & $229 / 51$ & $0.839(0.569-1.240)$ & 0.378 \\
\hline Bone & $29 / 13$ & $29 / 11$ & $0.449(0.195-1.034)$ & 0.06 \\
\hline Brain & $16 / 3$ & $16 / 10$ & $0.117(0.014-0.955)$ & 0.045 \\
\hline Liver & $s / 1$ & $5 / 1$ & $1.000(0.063-15.988)$ & 1 \\
\hline Lung & $21 / 5$ & $21 / 6$ & $0.090(0.017-0.470)$ & 0.004 \\
\hline Multiple & $46 / 21$ & $46 / 16$ & $0.574(0.292-1.126)$ & 0.106 \\
\hline
\end{tabular}

\section{Figures}



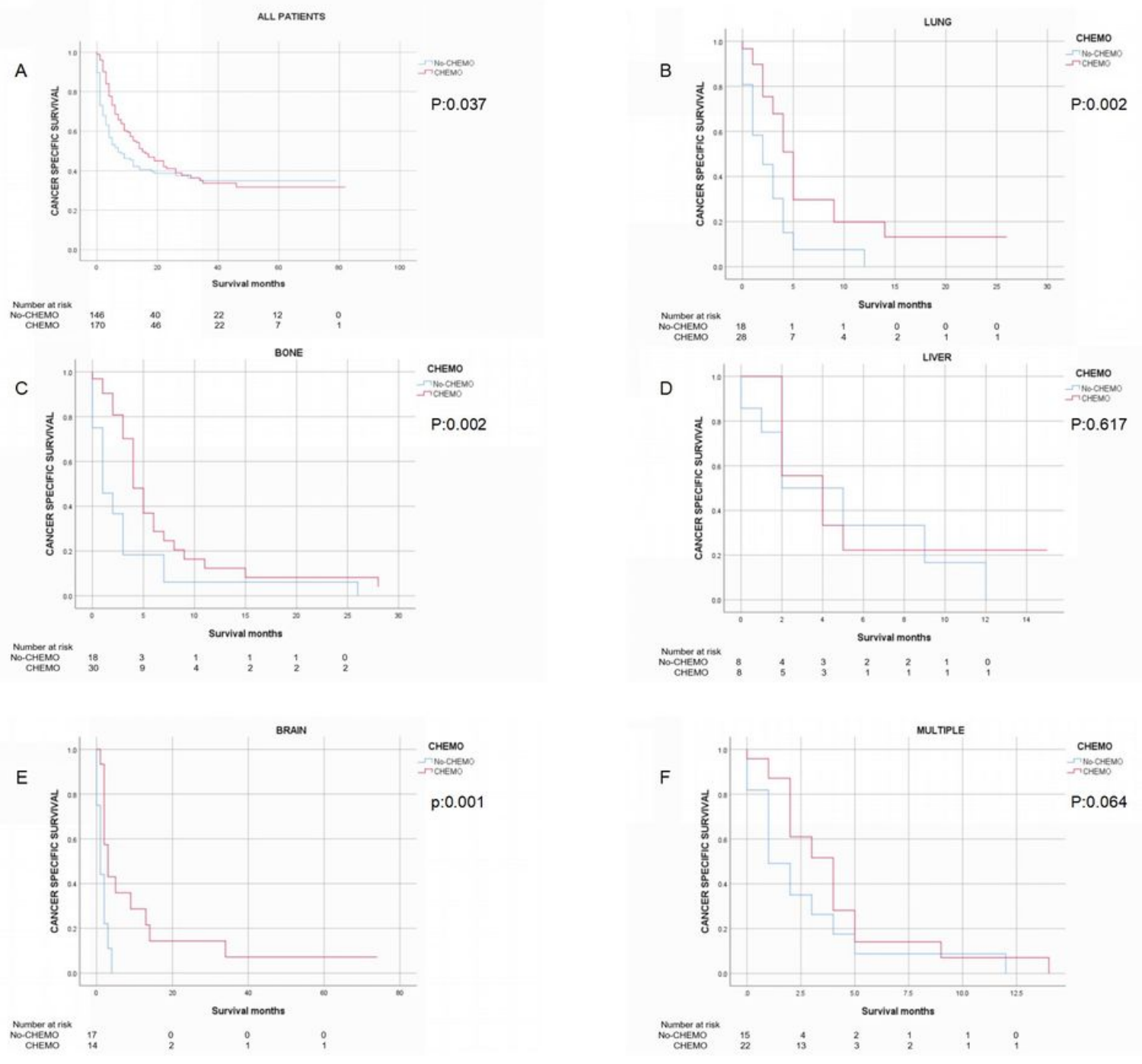

\section{Figure 1}

(A) Survival of GCLC Patients stratified by chemotherapy; (B) Survival of GCLC Patients with lung metastasis stratified by chemotherapy; (C) Survival of GCLC Patients with bone metastasis stratified by chemotherapy; (D) Survival of GCLC Patients with liver metastasis stratified by chemotherapy; (E) Survival of GCLC Patients with brain metastasis stratified by chemotherapy; (F) Survival of GCLC Patients with multiple metastasis stratified by chemotherapy 


\begin{tabular}{|c|c|c|c|}
\hline $\begin{array}{l}\text { Subgroup } \\
\text { Age }\end{array}$ & Chemo(patients/events) & No Chemo(patients/events) & Hazard Ratio(95\% Cl) \\
\hline Less than $71 \mathrm{yr}$ & $186 / 53$ & $186 / 54$ & $0.655(0.447-0.958)$ \\
\hline More than $71 \mathrm{yr}$ & $160 / 40$ & $160 / 41$ & $0.891(0.576-1.378)$ \\
\hline \multicolumn{4}{|l|}{ Race } \\
\hline White & $289 / 77$ & $289 / 71$ & $0.796(0.576-1.100)$ \\
\hline Black & $40 / 11$ & $40 / 19$ & $0.835(0.397-1.757)$ \\
\hline Other & $17 / 5$ & $17 / 5$ & $0.410(0.116-1.448)$ \\
\hline \multicolumn{4}{|l|}{ Sex } \\
\hline Male & $205 / 57$ & $205 / 59$ & $0.785(0.545-1.130)$ \\
\hline Female & $141 / 36$ & $141 / 36$ & $0.681(0.428-1.082)$ \\
\hline \multicolumn{4}{|l|}{ Grade } \\
\hline$|+1|$ & $6 / 2$ & $=-1$ & $0.744(0.559-0.991)$ \\
\hline III+IV & $340 / 91$ & $340 / 95$ & $0.726(0.544-0.969)$ \\
\hline \multicolumn{4}{|l|}{$\mathbf{T}$} \\
\hline T1 & $25 / 2$ & $25 / 2$ & $1.741(0.243-12.445)$ \\
\hline T2 & $113 / 30$ & $113 / 23$ & $0.983(0.577-1.713)$ \\
\hline T3 & $129 / 35$ & $129 / 46$ & $0.631(0.405-0.982)$ \\
\hline T4 & $79 / 26$ & $79 / 24$ & $0.310(0.170-0.566)$ \\
\hline \multicolumn{4}{|l|}{$\mathbf{N}$} \\
\hline No & $184 / 36$ & $184 / 37$ & $0.929(0.587-1.471)$ \\
\hline N1 & $45 / 18$ & $45 / 10$ & $0.827(0.379-1.807)$ \\
\hline N2 & $86 / 29$ & $86 / 34$ & $0.350(0.207-0.592)$ \\
\hline N3 & $31 / 10$ &  & $0.305(0.123-0.759)$ \\
\hline \multicolumn{4}{|l|}{ Surge } \\
\hline Yes & $143 / 27$ & $143 / 27$ & $0.853(0.500-1.455)$ \\
\hline No & 203/66 & $203 / 68$ & $0.599(0.426-0.843)$ \\
\hline \multicolumn{4}{|l|}{ Radio } \\
\hline Yes & $120 / 39$ & $120 / 39$ & $0.836(0.536-1.305)$ \\
\hline No & $226 / 54$ & $226 / 56$ & $0.699(0.480-1.017)$ \\
\hline \multicolumn{4}{|l|}{ Met } \\
\hline None & $229 / 50$ & $229 / 51$ & $0.839(0.569-1.240)$ \\
\hline Bone & $29 / 13$ & 29/11 & $0.449(0.195-1.034)$ \\
\hline Brain & $16 / 3$ & $16 / 10$ & $0.117(0.014-0.955)$ \\
\hline Liver & $5 / 1$ & $5 / 1$ & $11.000(0.063-15.988)$ \\
\hline Lung & $21 / 5$ & 21/6 & $0.090(0.017-0.470)$ \\
\hline Multiple & $46 / 21$ & $\mapsto-1$ & $0.574(0.292-1.126)$ \\
\hline
\end{tabular}

\section{Figure 2}

The forest plot for GCLC patients comparing CSS between the CHEMO group and no-CHEMO group according to different variable 
All Select None no plot density box Piot type
spikes
boxplot ecdt bars violin bean

$$
\text { Points }
$$

$\square \mathrm{T}$

$\square \mathrm{N}$

$\square \quad$ Metastasis

$\square \quad$ Surgery

$\square \quad$ Chemotherapy

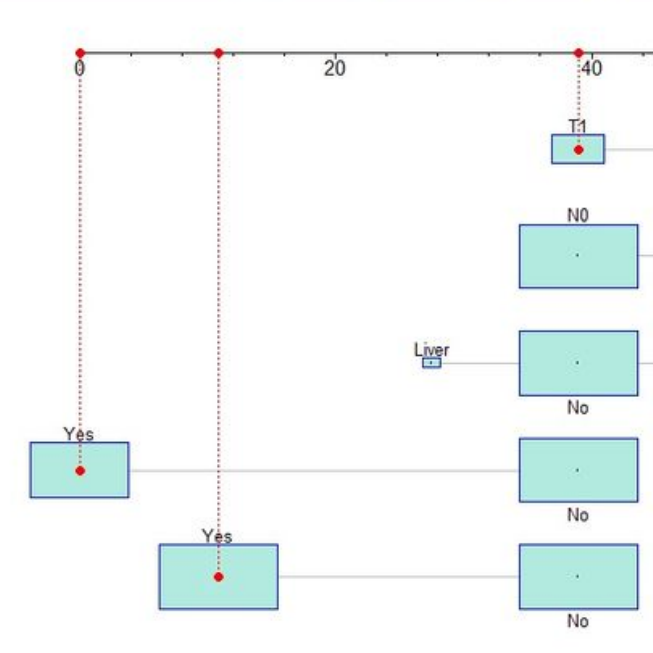

Total points

cox cph

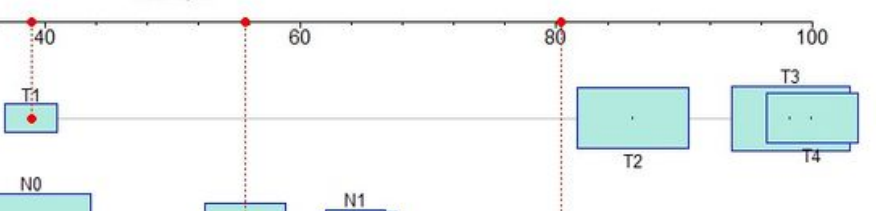

$\square$

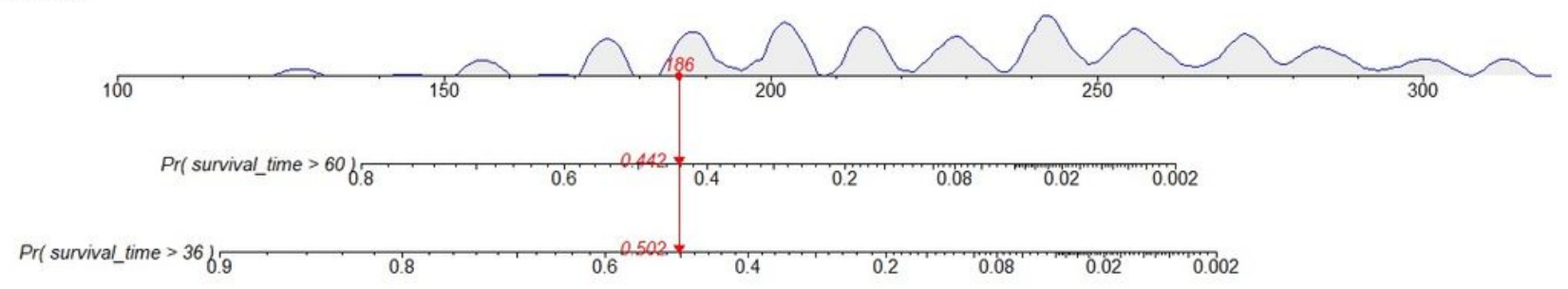

\section{Figure 3}

Cancer specific survival nomogram for GCLC patients.

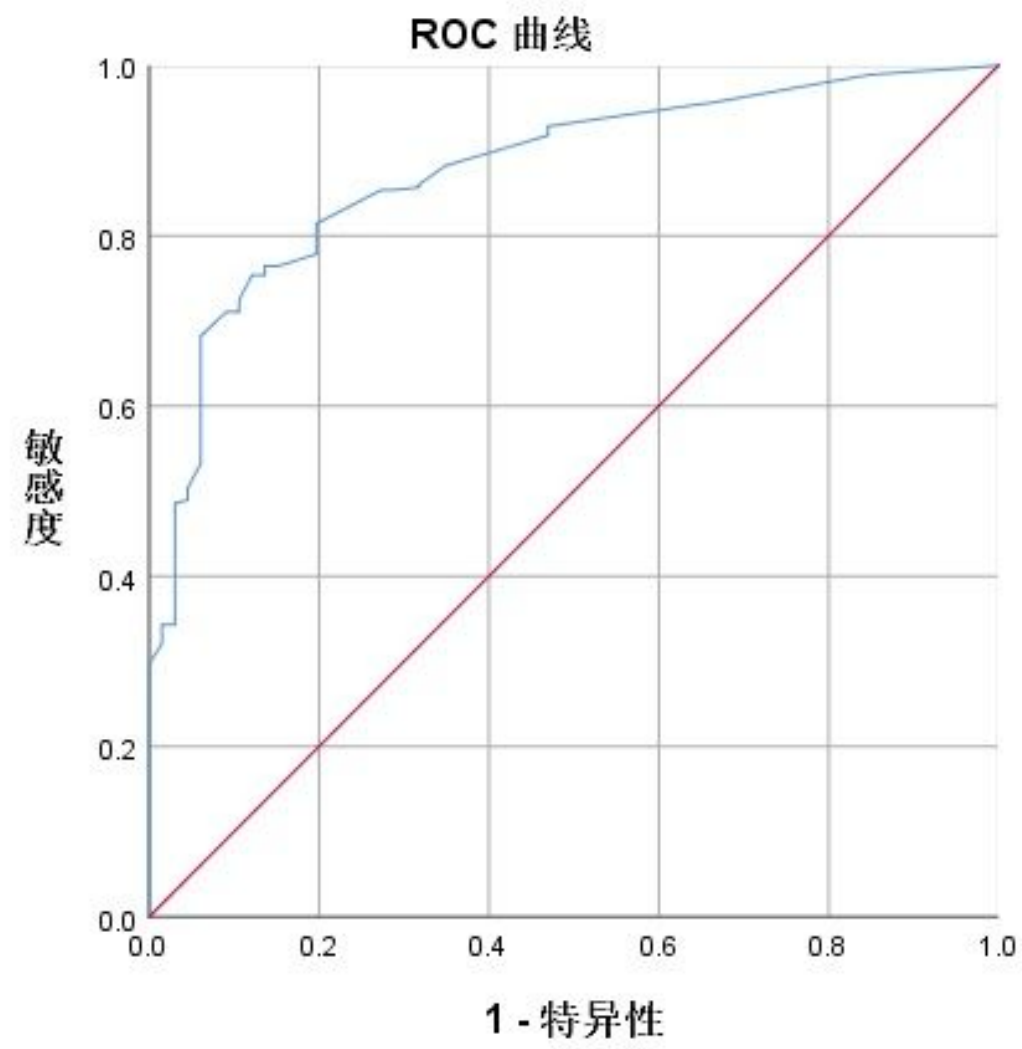

对角段由绑定值生成。 
Figure 4

Receiver operating characteristic (ROC) curves of clinical events in GCLC patients.

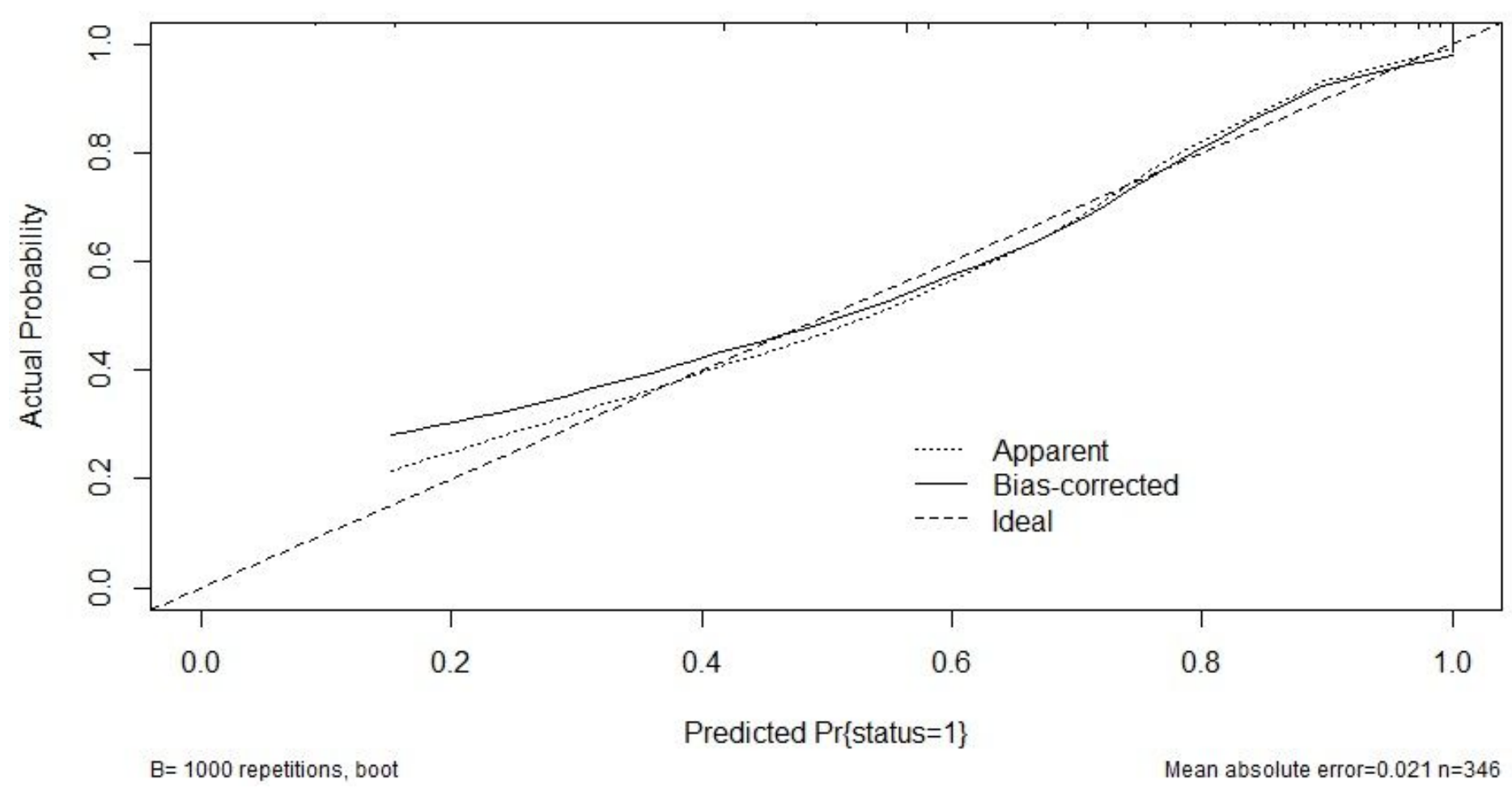

Figure 5

Calibration plot for the cancer specific survival nomogram. 